\title{
Ovarian Torsion
}

National Cancer Institute

\section{Source}

National Cancer Institute. Ovarian Torsion. NCI Thesaurus. Code C99084.

Twisting of the ovary resulting in the cutoff of the blood supply to the ovary. It may develop in an enlarged or normal ovary and in the vast majority of cases is unilateral. Signs and symptoms include acute lower abdominal pain, nausea, vomiting, and fever. 